Received: 2020/12/07, Revised: 2020/12/17, Accepted: 2020/12/22, Published: 2020/12/31 @2020 In-Soo Lim; Licence Physical Activity and Nutrition. This is an open access article distributed under the terms of the creative commons attribution license (https://creativecommons.org/licenses/by-nc/2.0/), which permits unrestricted use, distribution, and reproduction in any medium, provided the orginal work is properly cited.

${ }^{*}$ Corresponding author : In-Soo Lim, Ph.D.

Office of Academic Affairs, Changwon National University, 20 Changwondaehak-ro, Uichang-gu, Changwon, Gyeongnam 641-773, Republic of Korea.

Tel: +82-55-213-3525

@2020 The Korean Society for Exercise Nutrition
E-mail: iminsu@hanmail.net

\section{Effects of branched-chain amino acid supplement on knee peak torque and indicators of muscle damage following isokinetic exercise-induced delayed onset muscle soreness}

\author{
In-Soo Lim ${ }^{1 *}$ \\ 1. Department of Physical Education, Changwon National University, Changwon, Republic of Korea
}

\section{INTRODUCTION}

[Purpose] This study aimed to investigate the effects of branched-chain amino acid (BCAA) supplement on delayed onset muscle soreness (DOMS) by analyzing the maximum muscle strength and indicators of muscle damage.

[Methods] Twelve men with majors in physical education were assigned to the BCAA group and placebo group in a double-blinded design, and repeated measurements were conducted. DOMS was induced with an isokinetic exercise. Following BCAA administration, the changes in the knee extension peak torque, flexion peak torque, aspartate aminotransferase (AST), creatine kinase (CK), and lactate dehydrogenase (LDH) concentrations were analyzed. The maximum knee muscle strength was measured at the baseline (pre-D0) following BCAA administration for 5 days before exercise (-D5, -4D, -3D, -2D, -1D). In contrast, the post-treatment measurements (D3) were recorded after BCAA administration for 3 days (post-D0, D1, D2). Blood samples were obtained before (pre-D0), immediately after (post-D0), $24 \mathrm{~h}$ (D1), $48 \mathrm{~h}$ (D2), and $72 \mathrm{~h}$ (D3) after the exercise to analyze the indicators of muscle strength. BCAA was administered twice daily for 8 days ( 5 days and 3 days before inducing DOMS and during the experimental period, respectively).

[Results] There was no difference in the flexion peak torque between the groups. However, the BCAA group showed a significantly higher extension peak torque at D3 (second isokinetic exercise), compared to the placebo group $(p<.05)$. There was no difference in AST changes between the groups. Nonetheless, the CK and LDH were significantly reduced in the BCAA group, compared to the placebo group. There was no correlation between the extension peak torque and flexion peak torque. However, the CK and LDH increased proportionately in DOMS. Moreover, their concentrations significantly increased with a decreasing peak torque $(\mathrm{p}<.01)$.

[Conclusion] An exercise-induced DOMS results in a decrease in the peak torque and a proportional increase in the $\mathrm{CK}$ and LDH concentrations. Moreover, the administration of BCAA inhibits the reduction of the extension peak torque and elevation of $\mathrm{CK}$ and $\mathrm{LDH}$ concentrations. Therefore BCAA might be administered as a supplement to maintain the muscle strength and prevent muscle damage during vigorous exercises that may induce DOMS in sports settings.

[Key words] BCAA, DOMS, knee peak torque, indicators of muscle damage, AST, CK, LDH
Exercise-induced muscle damage is categorized into two types, namely acute muscle soreness and delayed onset muscle soreness (DOMS). DOMS occurs 8-10 hours after performing an exercise, with symptoms of pain appearing at 24-28 h. These symptoms alleviate 5-7 days later and are lost 8-10 days later. ${ }^{1,2}$ Exercise-induced DOMS diminishes athletic performance by reducing the range of motion and maximum muscle strength and might lead to musculoskeletal and neurological disorders. ${ }^{3,4}$ DOMS is assessed based on indicators, such as lactate, ammonia, myoglobin, lactate dehydrogenase (LDH), creatine kinase (CK), and aspartate aminotransferase (AST). CK is a typical enzyme used to determine musculoskeletal injury, where the CK levels continuously rise after a vigorous exercise. LDH is an oxidation-reduction enzyme that proportionately increases with exercise intensity. Furthermore, it increases more than twice after a prolonged endurance exercise. ${ }^{5}$ AST and alanine aminotransferase (ALT) are particularly used as indicators of hepatocellular damage after a prolonged vigorous exercise. While ALT is present in the liver cells, AST is found in the heart, liver, and skeletal muscles. AST leaks from the muscles to the blood upon a muscle injury. An elevated AST concentration is an indicator of muscle injury. ${ }^{6}$

Therapeutic interventions, such as stretching, massage, vibration, ultrasound, hyperthermia, cold therapy, acupuncture, microcurrent electrical therapy, and nutrition therapy have been introduced to alleviate DOMS symptoms. ${ }^{7-10}$ Moreover, effects of branched chain amino acids (BCAA) in muscle tissue regeneration and restoration as a nutrition therapy have particularly gained attention in recent years. BCAA is the primary essential amino acid component of muscle proteins and comprises a group of three amino acids, including leucine, isoleucine, and valine that are oxidized in the skeletal muscles. ${ }^{11}$ BCAA stimulates muscle tissue recovery and regeneration. Studies have reported on a decrease in exerciseinduced muscle injury by BCAA consumption. BCAA can increase the endurance capacity during an exercise by delaying muscle fatigue and reduces muscle pain. ${ }^{12-14}$ Few studies have investigated changes in the 
muscle strength and the indicators of muscle injury ${ }^{19-21}$ with BCAA administration. ${ }^{15-18}$ However, there are limited studies on the maximum muscle strength and the indicators of DOMS-induced muscle damage to examine the effects of BCAA. Thus, this study aimed to analyze the maximum muscle strength and indicators of muscle damage to examine the effects of BCAA on DOMS. In other words, we first induced DOMS using an isokinetic exercise and analyzed the changes of knee peak torques and blood AST, CK, and LDH after BCAA administration.

\section{METHODS}

\section{Participants}

Twelve men with majors in physical education from $\mathrm{C}$ University were enrolled. They were assigned to the BCAA and placebo group in a double-blinded method. We recorded repeated measurements with a 6 -week interval between the groups. The participants were healthy individuals without any clinical disease. All participants provided their signed informed consent. The mean age was $23 \pm 1.4$ years, with a mean height of $175.7 \pm 3.2 \mathrm{~cm}$ and a mean body weight of $73.2 \pm 5.2 \mathrm{~kg}$.

\section{Experimental procedures}

Experimental design is shown in Figure 1. We measured the knee extension peak torque and flexion peak torque, before and after BCAA administration, before and after inducing DOMS. More specifically, the first isokinetic exercise measurement was recorded (D0) after administering BCAA for 5 days before an exercise (-D5, -4D, -3D, -2D, and -1D). In contrast, we recorded the second isokinetic exercise measurements (D3) after administering BCAA for 3 days (D0, D1, and D2) following an exercise to induce DOMS. Furthermore, we obtained blood samples before (pre-D0), immediately after (post-D0), and $24 \mathrm{~h}$ (D1), $48 \mathrm{~h}$ (D2), and $72 \mathrm{~h}$ (D3) after the exercise. This helped us analyze changes in the indicators of muscle injury based on BCAA administration, before and after inducing DOMS.

\section{Exercise-induced DOMS}

DOMS was induced by performing an isokinetic exercise using an isokinetic testing system (Cybex CSMi Humac NORM, 770), based on the methods proposed by Cornish \& Johnson. ${ }^{22}$ In other words, the participants performed six sets (10 repetitions per set) of isokinetic exercise at $60 \%$ of the knee extension peak torque at $120 \% \mathrm{sec}$ angular velocity. They were provided a break for $10 \mathrm{~s}$ between the sets. We recorded the measurements between 9-11:00 am at the pretreatment baseline (D0), following 5 days of BCAA administration, and $72 \mathrm{~h}$ after the exercise (D3). We measured the knee extension peak torque and flexion peak torque.

\section{BCAA supplement}

BCAA supplements were administered twice daily from 5 days before the exercise to induce DOMS (-D5) and for $72 \mathrm{~h}$ after the exercise (3D), for a total of 8 days. AMINOVITAL 400 (A, Japan) was used as the supplement. They were consumed before breakfast and lunch. Each pouch (total calorie $18.7 \mathrm{Kcal}$ ) contains $3 \mathrm{~g}, 4.0 \mathrm{~g}$, and $1 \mathrm{mg}$ of BCAA, protein (leucine $1.60 \mathrm{~g}$, isoleucine $0.43 \mathrm{~g}$, valine $0.44 \mathrm{~g}$, and other amino acids $1.51 \mathrm{~g}$ ), and vitamin B, respectively. Considering the maximum recommended dose of $1.6 \mathrm{~g} / \mathrm{kg} \mathrm{BCAA}, 0.6 \mathrm{~g} / \mathrm{kg}$ of BCAA was consumed in the morning and afternoon after diluting it in water. The placebo was packaged to conceal the contents from the participants. A similar amount was diluted in orange juice and administered in the morning and afternoon at the same time as the BCAA. All participants were requested to maintain a diet journal during the experiment.

\section{Blood analyses}

Blood samples $(10 \mathrm{ml})$ were collected between 8-9:00 am in the morning in a fasted state from the brachial vein. The collected samples were clotted for $30 \mathrm{~min}$ at room temperature and centrifuged for $10 \mathrm{~min}$ at $3,000 \mathrm{rpm}$ to separate the serum. We stored the separated serum samples in a freezer at $-90^{\circ} \mathrm{C}$. We used Modular Analytics (Roche, Germany) to analyze the blood CK, LDH, and AST concentrations.

\section{Statistical analyses}

The mean (M) and standard deviation (SD) were computed for each parameter using the SPSS software (version, $20)$. We conducted two-way analysis of variance with

- BCAA supplement

$\downarrow$ Blood sampling

Isokinetic exercise

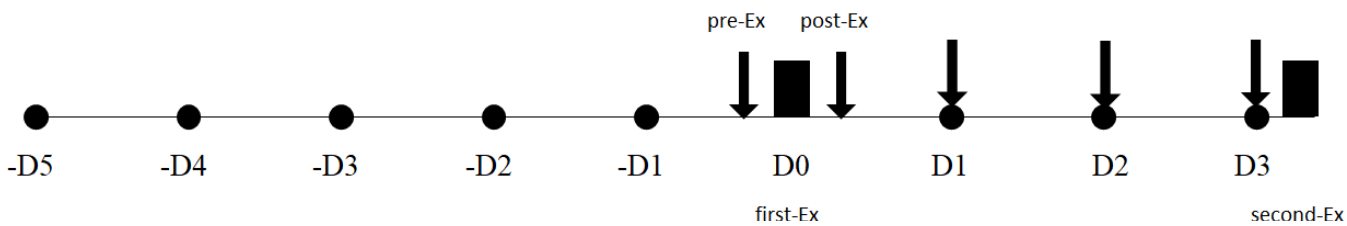


repeated measures to analyze the significance of the data according to the treatment and time of measurement. A post-hoc comparison was performed using independent and dependent $\mathrm{t}$-tests according to the time of measurement. The statistical significance was set at $\mathrm{P}<.05$. The Pearson's correlation method was used to analyze the correlations between the peak torque and the indicators of muscle injury.

\section{RESULTS}

\section{Knee extension and flexion peak torque}

Figures 2 and 3 show the changes in knee extension and flexion peak torque between two groups. The placebo group demonstrated a significant reduction of the extension peak torque from $332.3 \pm 34 \mathrm{Nm}$ at the baseline (D0) to $267.3 \pm 28$ $\mathrm{Nm}$ during post-treatment (D3) $(\mathrm{p}<.05)$. The BCAA group displayed a reduction of the extension peak torque from $355.6 \pm 43 \mathrm{Nm}$ at the baseline to $313.2 \pm 51 \mathrm{Nm}$ at the post-treatment. However, the change was insignificant. There were significant differences between the groups. The post-hoc test revealed a significant reduction in the placebo group, compared to the BCAA group $(\mathrm{p}<.05)$. Regarding flexion peak torque, The placebo group revealed a significant reduction in the flexion peak torque from $188.3 \pm 27$ $\mathrm{Nm}$ at the baseline (D0) to $143.2 \pm 26 \mathrm{Nm}$ at post-treatment (D3) $(\mathrm{p}<.05)$. In contrast, the BCAA group displayed an insignificant reduction from $191.2 \pm 36 \mathrm{Nm}$ at the baseline to $177.5 \pm 24 \mathrm{Nm}$ at post-treatment.

\section{Blood AST}

Figure 4 shows the changes in blood AST between two groups. The placebo group showed a significant elevation in the AST concentration from 13.7 $\pm 3.2 \mathrm{U} / \mathrm{L}$ before the exercise (pre-D0) to $25.1 \pm 3.8 \mathrm{U} / \mathrm{L}, 39.2 \pm 4.8 \mathrm{U} / \mathrm{L}, 48.4 \pm 6.7 \mathrm{U} /$ $\mathrm{L}$, and $36.3 \mathrm{U} \pm 7.2 \mathrm{U} / \mathrm{L}$ immediately after (post-D0), and at $24 \mathrm{~h}$ (D1), $48 \mathrm{~h}$ (D2), and $72 \mathrm{~h}$ (D3) after the exercise, respectively $(\mathrm{p}<.05)$. Further tests showed that the AST levels

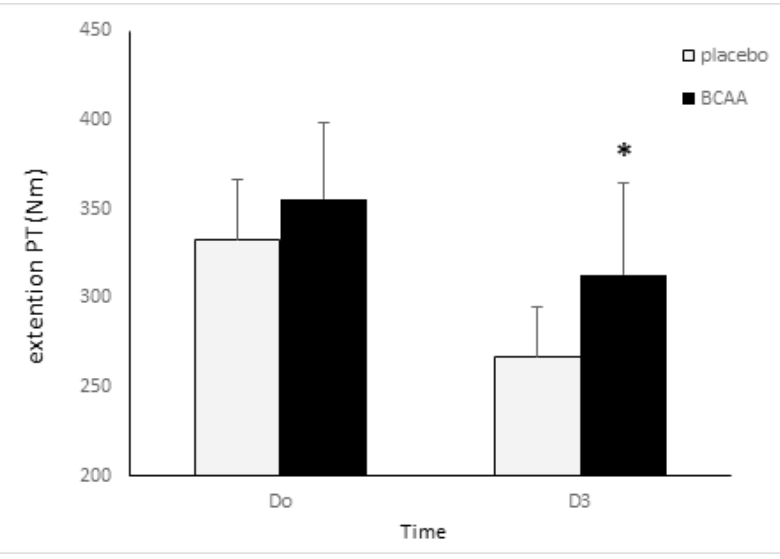

Figure 2. A comparison of the knee extension PT following an exercise-induced DOMS between the BCAA and placebo group.

PT: peak torque; BCAA: branched-chain amino acid; D0: immediately after exercise; D3: $3 \mathrm{~d}$ after exercise * $p<.05$. had significantly increased after the exercise (post-D0, D1, D2, and D3), compared to the pre-exercise concentrations (pre-D0). In addition, the levels were significantly elevated at $\mathrm{D} 2$, compared to that at post-D0. The BCAA group revealed a substantial increase in the AST levels from $15.2 \pm 3.1$ $\mathrm{U} / \mathrm{L}$ before the exercise (pre-D0) to $27.6 \pm 4.8 \mathrm{U} / \mathrm{L}, 37.5 \pm 6.8$ $\mathrm{U} / \mathrm{L}, 42.7 \pm 7.2 \mathrm{U} / \mathrm{L}$, and $33.4 \pm 5.9 \mathrm{U} / \mathrm{L}$ immediately after (post-D0), and at $24 \mathrm{~h}$ (D1), $48 \mathrm{~h}$ (D2), and $72 \mathrm{~h}$ (D3) after the exercise, respectively $(\mathrm{p}<.05)$. Further tests showed that the AST levels had significantly increased after the exercise (post-D0, D1, D2, and D3), compared to the pre-exercise concentrations (pre-D0) $(\mathrm{p}<.05)$. Moreover, the levels were significantly elevated at D2, compared to that at post-D0 $(p<.05)$. There were no significant differences between the groups.

\section{Blood CK}

Figure 5 shows the changes in blood $\mathrm{CK}$ between two

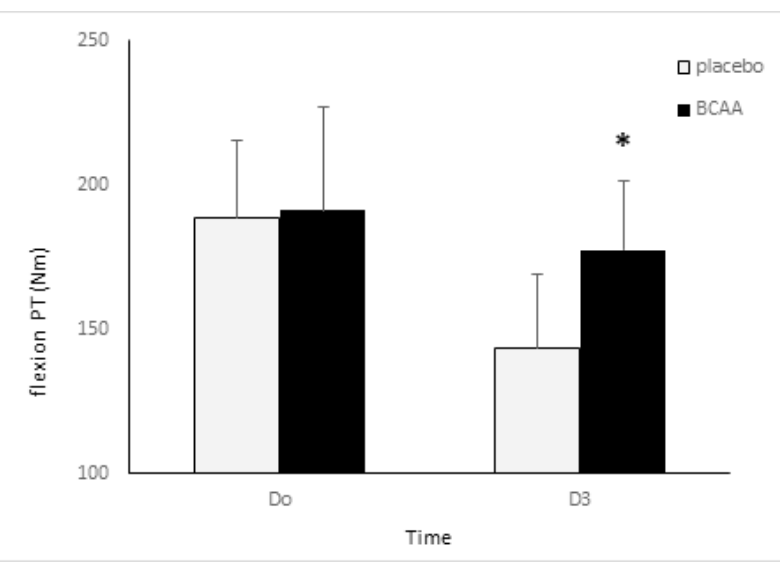

Figure 3. A comparison of the flexion PT following an exercise-induced DOMS between the BCAA and placebo group. PT: peak torque; BCAA: branched-chain amino acid; D0: immediately after exercise; D3: $3 \mathrm{~d}$ after exercise. ${ }^{*} p<.05$.

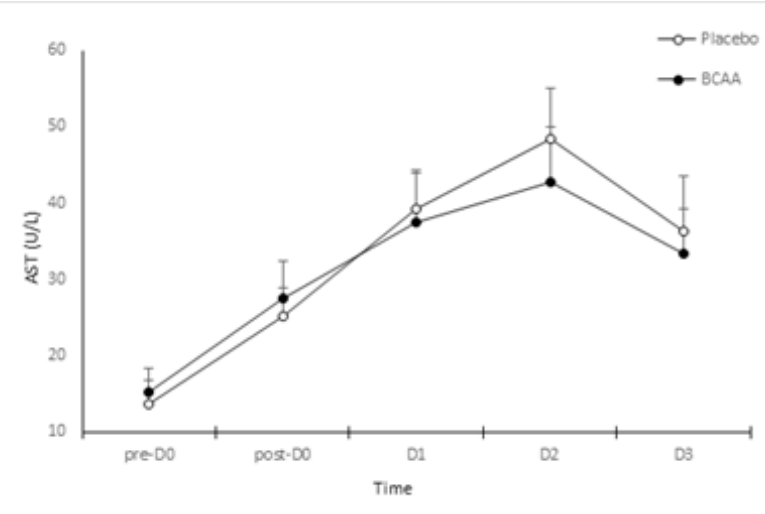

Figure 4. A comparison of changes in blood AST levels following an exercise-induced DOMS between the BCAA and placebo group. BCAA: branched-chain amino acid; AST: aspartate aminotransferase; Pre-D0: pre exercise; Post-D0: immediately after exercise; D1: $24 \mathrm{~h}$ after exercise; D2: $2 \mathrm{~d}$ after exercise; D3: $3 \mathrm{~d}$ after exercise. ${ }^{*} \mathrm{p}<.05$. 
groups. The CK concentration significantly and continuously increased in the placebo group from $157.3 \pm 23.8 \mathrm{U} /$ $\mathrm{L}$ before the exercise (pre-D0) to $221.4 \pm 36.1 \pm 36.1 \mathrm{U} / \mathrm{L}$, $281.7 \pm 42.8 \mathrm{U} / \mathrm{L}, 366.3 \pm 51.7 \mathrm{U} / \mathrm{L}$, and $382.8 \pm 47.2 \mathrm{U} / \mathrm{L}$ immediately after (post-D0), and at $24 \mathrm{~h}$ (D1), $48 \mathrm{~h}$ (D2), and $72 \mathrm{~h}$ (D3) after the exercise, respectively $(\mathrm{p}<.05)$. Further tests showed that the CK levels had significantly increased after the exercise (post-D0, D1, D2, and D3), compared to the pre-exercise concentrations (pre-D0). In addition, the levels were significantly elevated at D1 and D2, compared to that at post-D0 $(\mathrm{p}<.05)$. The BCAA group displayed a continuous and significant increase in the CK concentrations from $141.3 \pm 17.1 \mathrm{U} / \mathrm{L}$ before the exercise (pre-D0) to $236.2 \pm 34.8 \mathrm{U} / \mathrm{L}, 289.3 \pm 36.8 \mathrm{U} / \mathrm{L}, 304.5 \pm 47.2 \mathrm{U} / \mathrm{L}$, and $314.8 \pm 37.9 \mathrm{U} / \mathrm{L}$ immediately after (post-D0), and at $24 \mathrm{~h}$ (D1), $48 \mathrm{~h}$ (D2), and $72 \mathrm{~h}$ (D3) after the exercise, respectively $(p<.05)$. Further tests showed that the CK levels had significantly increased after the exercise (post-D0, D1, D2, and D3), compared to the pre-exercise concentrations (pre-D0). Furthermore, the levels were significantly elevated at D1, $\mathrm{D} 2$, and D3, compared to that at post-D0 $(\mathrm{p}<.05)$. There were significant differences among the groups. The post-hoc comparison revealed substantially elevated CK levels in the placebo group at $\mathrm{D} 2$ and $\mathrm{D} 3$, compared to that in the BCAA group $(\mathrm{p}<.05)$.

\section{Blood LDH}

Figure 6 shows the changes in blood LDH between two groups. The placebo group revealed a significant increase in the LDH levels from $142.3 \pm 23 \mathrm{U} / \mathrm{L}$ before exercise (pre-D0) to $234.4 \pm 43 \mathrm{U} / \mathrm{L}, 279.7 \pm 48 \mathrm{U} / \mathrm{L}, 326.3 \pm 41 \mathrm{U} / \mathrm{L}$, and $287.8 \pm 37 \mathrm{U} / \mathrm{L}$ immediately after (post-D0), and at $24 \mathrm{~h}$ (D1), $48 \mathrm{~h}$ (D2), and $72 \mathrm{~h}$ (D3) after the exercise, respectively $(p<.05)$. Further tests showed that the CK levels had significantly increased after the exercise (post-D0, D1, D2, and D3), compared to the pre-exercise concentrations (pre-D0). Furthermore, the levels were significantly elevated at D2,

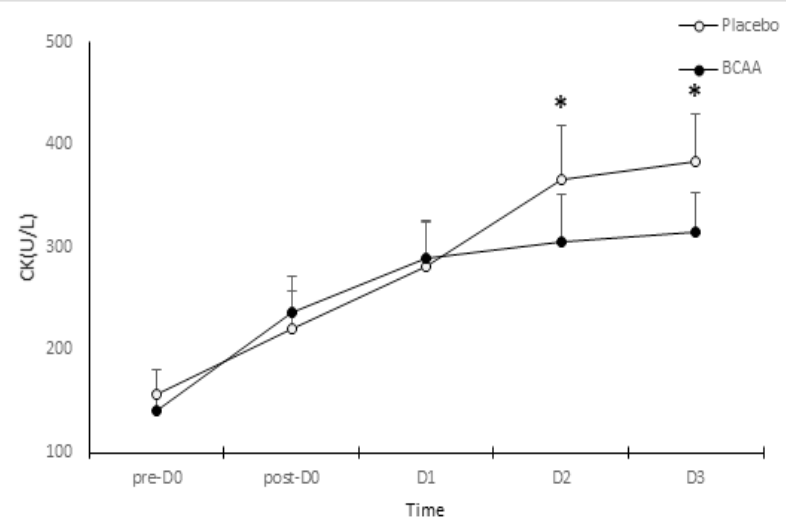

Figure 5. A comparison of changes in blood CK levels following an exercise-induced DOMS between the BCAA and placebo group. BCAA: branched-chain amino acid; CK: creatine kinase; Pre-D0: pre exercise. Post-D0: immediately after exercise; D1: 24 h after exercise; D2: 2 d after exercise; D3: 3 d after exercise. ${ }^{*} p<.05$ compared to that at post-D0 $(\mathrm{p}<.05)$. LDH levels in the BCAA group had significantly changed from $153.3 \pm 18 \mathrm{U} /$ L before the exercise (pre-D0) to $221.2 \pm 48 \mathrm{U} / \mathrm{L}, 273.3 \pm 56$ $\mathrm{U} / \mathrm{L}, 298.5 \pm 37 \mathrm{U} / \mathrm{L}$, and $244.8 \pm 32 \mathrm{U} / \mathrm{L}$ immediately after (post-D0), and at $24 \mathrm{~h}$ (D1), $48 \mathrm{~h}$ (D2), and $72 \mathrm{~h}$ (D3) after the exercise, respectively $(\mathrm{p}<.05)$. Further tests showed that the CK levels had significantly increased after the exercise (post-D0, D1, D2, and D3), compared to the pre-exercise concentrations (pre-D0). Moreover, the levels were significantly elevated at D2, compared to that at pre-D0 $(\mathrm{p}<.05)$. There were significant differences between the groups. The post-hoc comparison revealed substantially elevated LDH levels at D2 and D3 in the placebo group, compared to that in the BCAA group $(\mathrm{p}<.05)$.

The correlation between the peak torque and indicators of muscle damage

Table 1 summarizes correlations between the extension and flexion peak torques and the levels of $\mathrm{CK}, \mathrm{LDH}$, and ASK in the BCAA group on the last day of measurement (D3).

There was no correlation between the extension and flexion peak torques. The extension peak torque was negatively correlated with the CK and LDH levels. In other words, the CK and LDH levels decreased with an increasing extension

Table 1. Correlations between the knee PT, CK, LDH, and ASK.

\begin{tabular}{c|c|cccc} 
& Extension & Flexion & CK & LDH & AST \\
\hline Extension & & .185 & $-.906^{* *}$ & $-.733^{* *}$ & -.069 \\
Flexion & & -.213 & $-.647^{*}$ & -.566 \\
CK & & & $.692^{*}$ & .153 \\
LDH & & & & $.620^{*}$ \\
AST & & & &
\end{tabular}

PT: peak torque; AST: aspartate aminotransferase; CK: creatine kinase; LDH: lactate dehydrogenase. ${ }^{*} p<.05 ;{ }^{* *} p<.01$.

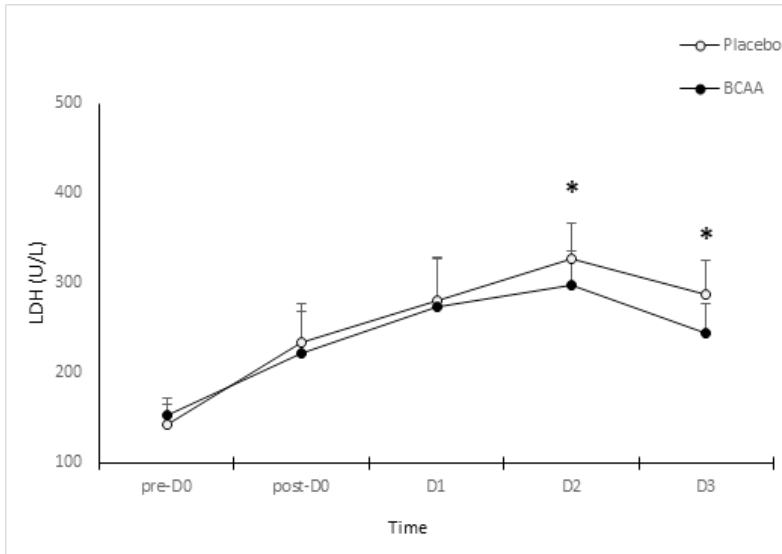

Figure 6. A comparison of changes in blood LDH levels following an exercise-induced DOMS between the BCAA and placebo group. BCAA: branched-chain amino acid; LDH: lactate dehydrogenase; Pre-D0: pre exercise; post-D0: immediately after exercise; D1: $24 \mathrm{~h}$ after exercise; D2: $2 \mathrm{~d}$ after exercise; D3: $3 \mathrm{~d}$ after exercise. ${ }^{*} p<.05$ 
peak torque $(\mathrm{p}<.05)$. Furthermore, the flexion peak torque was negatively correlated with the LDH level. The latter decreased with an increasing flexion peak torque $(p<.05)$. The $\mathrm{CK}$ and LDH levels were positively correlated, thus indicating the levels proportionately changed to each other $(\mathrm{p}<.05)$.

\section{DISCUSSION}

Both groups showed a significant reduction in the extension peak torque at the second isokinetic exercise (D3), compared to that at the baseline because of the DOMS. The BCAA group had a significantly higher value at D3, compared to the placebo group. Furthermore, both groups showed a significant reduction in the flexion peak torque at the D3, compared to that at D0. In addition, the BCAA group had a significantly higher value, compared to the placebo group. A prior study ${ }^{23}$ reported on a substantial increase in the peak torque after inducing DOMS with resistance exercise in the BCAA group, compared to the placebo group. Furthermore, following the isokinetic exerciseinduced DOMS, the peak torque decreased until $96 \mathrm{~h}$ after the exercise. ${ }^{24}$ There was a decrease in the extension peak torque until 72 hours after the DOMS-inducing exercise. The BCAA supplements inhibited the reduction of the peak torque during the aforementioned process. Our results support previous findings that BCAA administration can inhibit the exercise-mediated breakdown of muscle proteins. ${ }^{25}$ Furthermore, Howatson et al. ${ }^{26}$ mentioned that the action of BCAA in stimulating protein synthesis facilitates the quick recovery of muscle injury caused by resistance exercise. This was attributed to the BCAA-controlled inhibition of the breakdown of skeletal muscle proteins. ${ }^{18}$ BCAA is particularly involved in escalated protein synthesis by stimulating the insulin-like growth factor 1 . This eventually stimulates the mammalian target of rapamycin (mTOR). ${ }^{27}$ Therefore, BCAA administration after a vigorous exercise might promote protein synthesis during the post-exercise recovery phase. The extension and flexion peak torques were higher in the BCAA group before and after DOMS. This might be a consequence of an increased protein synthesis because of BCAA administration, which in turn inhibited the reduction of peak torque.

The AST tended to increase after the exercise (postD0, D1, D2, and D3), compared to that before the exercise (pre-D0). Nonetheless, there were no significant differences between the two groups. The AST concentrations tended to increase until $48 \mathrm{~h}$ (D2) the after DOMS-inducing exercise. This supports previous findings that reported on significant elevations in ASK levels after a high-intensity training that induces muscle injury and muscle fatigue. ${ }^{28}$ However, BCAA administration did not reduce the ASK levels. The AST levels particularly increased until (D2). However, they began to decline after D3, unlike the trends observed for the CK and LDH levels. A prior study reported on an increase in the blood ASK levels by more than seven-fold in liver cells compared to that in the skeletal muscles during a marathon. ${ }^{29}$ Therefore, the ASK levels are more specific to liver cells than skeletal muscles in exercises the induce muscle injuries. In contrast, the CK and LDH concentrations tended to increase over time after a DOMS-inducing exercise. The BCAA group showed a significantly lower value of $\mathrm{CK}$ and $\mathrm{LDH}$, compared to the placebo group. According to a previous study, ${ }^{1}$ the $\mathrm{CK}$ levels begin to increase $24 \mathrm{~h}$ after a vigorous exercise and peak at $48 \mathrm{~h}$. Similarly, the CK and LDH levels significantly increased until $72 \mathrm{~h}$ (D3) after the exercise. Moreover, BCAA administration inhibited the increase of the CK and LDH levels. Our findings support the results of Greer et al. that BCAA administration inhibits muscle injuries caused by an endurance exercise by reducing the $\mathrm{CK}$ and LDH levels. ${ }^{20}$ This was attributed to the action of BCAA in reducing muscle protein oxidation. ${ }^{30}$ Rahimolou et al. ${ }^{31}$ conducted a meta-analysis on BCAA and muscle injuries and stated that BCAA inhibits muscle injuries. Therefore, their continuous use would be effective for endurance athletes. Furthermore, a prior study ${ }^{19}$ reported on a significant decline in the $\mathrm{CK}$ and $\mathrm{LDH}$ levels after administering BCAA for 7 days, followed by an endurance exercise. However, another study ${ }^{32}$ stated that BCAA administration prior to resistance exercise did not change the $\mathrm{CK}$ levels. The inconsistent results are reportedly affected by the type of exercise, intensity, duration, recovery period, and the duration and amount of BCAA administration. ${ }^{33,34}$ Exercise programs that may particularly induce DOMS can lead to different outcomes. There was no correlation between the extension and flexion peak torques. Therefore, BCAA administration did not affect the interaction between the agonistic and antagonistic muscles in the knee peak torque in a state of DOMS. In contrast, the extension peak torque was negatively correlated with the CK and LDH levels. In other words, the $\mathrm{CK}$ and $\mathrm{LDH}$ concentrations changed mutually and proportionately. The aforementioned levels decreased with an increasing extension peak torque. However, the LDH levels declined with an increasing flexion peak torque. In conclusion, while the peak torque decreases, the $\mathrm{CK}$ and LDH levels proportionately increase upon inducing DOMS by an exercise. However, BCAA inhibits the reduction of the extension peak torque and the elevation of CK and LDH levels during this process. Therefore, BCAA can be used as a supplement for athletes engaging in vigorous exercise. This might induce DOMS to maintain muscle strength and prevent muscle damage.

\section{ACKNOWLEDGEMENTS}

This research has been financially supported by Changwon National University in 2019-2021.

\section{REFERENCES}

1. Timon R, Tejero J, Brazo-Sayavera J, Carmen Crespo C, Olcina G. Effect of whole-body vibration after eccentric exercise on muscle soreness and muscle strength recovery. J Phys Ther Sci. 2016;28:1781-5.

2. Peake J, Nosaka K, Suzuki K. Attenuation of protective effect 
against eccentric exercise in humans. Exercise and Immunology Review. 2005;11:64-85.

3. Lieber RL, Friden J. Morphologic and mechanical basis of delayedonset muscle soreness. J Am Acad Orthop Surg. 2002;10:67-73.

4. Nosaka K, Newton M. Concentric or eccentric training effect on eccentric exercise-induced muscle damage. Medi Sci Sports Exer. 2002;34:59-63.

5. Kabayashi Y, Takeuchi T, Hosoi T, Yoshizaki H, Loeppky JA. Effect of a marathon run on serum lipprptein, CK, and LDH in recreational runners. Res Q Exerc Sports. 2005;76:450-5.

6. Barranco T, Tvarijonaviciute A, Tecles F, Carrillo JM, SánchezResalt $C$. Changes in creatine kinase, lactate dehydrogenase and aspartate aminotransferase in saliva samples after an intense exercise: a pilot study. J Sports Medi Physi Fitness. 2017;58:910-6.

7. Farr T, Nottle C, Nosaka K, Sacco P. The effects of therapeutic massage on delayed onset muscle soreness and muscle function following downhill walking. J Sci Med Sport. 2020;5:297-306.

8. Draper SN, Kullman EL, Sparks KE, Little K, Thoman J. Effects of intermittent pneumatic compression on Delayed Onset Muscle Soreness (DOMS) in long distance runners. Int J Exerc Sci. 2020;13:75-86.

9. Zainuddin Z, Hope P, Newton M, Sacco P, Nosaka K. Effects of partial immobilization after eccentric exercise on recovery from muscle damage. J Athletic Training. 2005;40:197-202.

10. Podbielski P. Effect of phototherapy on delayed onset muscle soreness. Photomedicine and Laser Surgery. 2006;24:377-82.

11. Holeček M. Branched-chain amino acids and branched-chain keto acids in hyperammonemic states: metabolism and as supplements. Metabolites. 2020;10:324.

12. Blomstrand E, Eliasson J, Karlsson HK, Kohnke R. Branchedchain amino acids activate key enzymes in protein synthesis after physical exercise. Journal of Nutrition. 2006;136:269-73.

13. Falavigna $\mathrm{G}$, Alves de Araújo J Jr, Rogero MM, Pires IS, Pedrosa RG, Martins E Jr, Alves de Castro I, Tirapegui J. Effects of diets supplemented with branched-chain amino acids on the performance and fatigue mechanisms of rats submitted to prolonged physical exercise. Nutrients. 2012;4:1767-80.

14. Jackman SR, Witard OC, Jeukendrup AE, Tipton KD. Branchedchain amino acid ingestion can ameliorate soreness from eccentric exercise. Medi Sci Sports Exer. 2010;42:962-70.

15. Matsumoto K, Koba T, Hamada K, Sakurai M, Higuchi T, Miyata H. Branched-chain amino acid supplementation attenuates muscle soreness, muscle damage and inflammation during an intensive training program. J Sports Medi Physi Fitness. 2009;49:424-31.

16. Rahimi MH, Shab-Bidar S, Mollahosseini M, Djafarian K. Branched-chain amino acid supplementation and exercise-induced muscle damage in exercise recovery: a meta-analysis of randomized clinical trials. Nutrition. 2017;42:30-6.

17. Shimomura Y, Yamamoto Y, Bajotto G, Sato J, Murakami T, Shimomura N, Kobayashi H, Mawatari K. Nutraceutical effects of branched-chain amino acids on skeletal muscle. J Nutr. 2006;136:529-32.

18. Busquets $\mathrm{S}$, Alvarez B, Llovera M, Agell N, López-Soriano FJ, Argilés JM. Branched-chain amino acids inhibit proteolysis in rat skeletal muscle: mechanisms involved. J Cell Physiol. 2000;184:380-4

19. Coombes JS, McNaughton LS. Effects of branched-chain amino acid supplementation on serum creatine kinase and lactate de- hydrogenase after prolonged exercise. J sports medicine physi fitness. 2000;40:240.

20. Greer BK, Woodard JL, White JP, Arguello EM, Haymes EM. Branched-chain amino acid supplementation and indicators of muscle damage after endurance exercise. Int J Sport Nutr Exer Metab. 2007:17:595-607.

21. Vatani DS, Abmadi S. Effect of oral Branched-chain amino acid supplementation prior to resistance exercise on metabolic hormones, plasma amino acid, and serum indices of muscle damage in the recovery period. Top Clin Nutr. 2016;31:346-54.

22. Cornish SM, Johnson ST. Systemic cytokine response to three bouts of eccentric exercise. Results Immunol. 2014;4:23-9.

23. Shimomura $Y$, Inaguma $A$, Watanabe $S$, Yamamoto $Y$, Muramatsu Y, Bajotto G, Sato J, Shimomura N, Kobayashi H, Mawatari K. Branched-chain amino acid supplementation before squat exercise and delayed-onset muscle soreness. Int J Sport Nutr Exerc Metab. 2010;20:236-44.

24. Eston R, Peters D. Effect of cold water immersion on the symptoms of exercise includes muscle damage. J Sports Sci. 1999;7:231-8.

25. MacLean DA, Graham TE, Saltin B. Branched-chain amino acids augment ammonia metabolism while attenuating protein breakdown during exercise. Am J Physiol. 1994;267:1010-22.

26. Howatson G, Hoad M, Goodall S, Tallent J, Bell PG, French DN. Exercise-induced muscle damage is reduced in resistance-trained males by branched chain amino acids: a randomized, double-blind, placebo controlled study. J Int Soc Sports Nutr. 2012;9:20.

27. Iresjö BM, Engström C, Smedh U, Lundholm K. Iresjö BM, Engström C, Smedh U, Lundholm K. Overnight steady-state infusions of parenteral nutrition on myosin heavy chain transcripts in rectus abdominis muscle related to amino acid transporters, insulin-like growth factor 1, and blood amino acids in patients aimed at major surgery. JPEN J Parenter Enteral Nutr. 2019;43:497-507.

28. Mashiko T, Umeda T, Nakaji S, Sugawara K. Effects of exercise on the physical condition of collage rugby players during summer training camp. Br J Sports Med. 2004;38:186-90.

29. Skenderi KP, Kavouras SA, Anastasiou CA, Yiannakouris N, matalas AL. Exertional rhabdomyolsis during a $246-\mathrm{km}$ continuous running race. Med Sci Sports Exerc. 2006;38:1054-7.

30. Haman F, Peronnet F, Kenny GP. Effects of carbohydrate availability on sustained shivering I. oxidation of plasma glucose, muscle glycogen, and protein. J Appl Physiol. 2004;96:32-40.

31. Rahimlou M, Ahmadi AHR, Palimi E, Mahdipour M, Poodeh BM. Reduction of muscle injuries and improved post exercise recovery by BCAA supplement: a systematic review and mata-analysis. $J$ Nutri Fasting Health. 2020;8:1-16.

32. Sheikholeslami-Vatani D, Ahmadi S. Effect of oral branched-chain amino acid supplementation prior to resistance exercise on metabolic hormones, plasma amino acids, and serum indices of muscle damage in the recovery period. Top Clin Nutr. 2016;31:346-54.

33. Lippi G, Schena F, Montagnana M, Slavagno GL, Benfi G, Guidi GC. Significant variation of traditional marker of liver injury after a half-marathon run. Eur J Intern Med. 2011;22:36-8.

34. Lippi G, Schena F, Montagnana M, Slavagno GL, Benfi G, Guidi GC. Acute variation of biochemicalmarker of muscle damage following a 21-km, half-marathon run. Scan J Clin Lab Invest. 2008;68:667-72. 